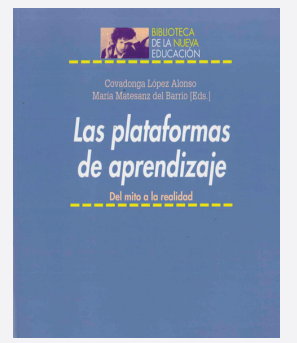

\title{
Las plataformas de aprendizaje: del mito a la realidad
}

\author{
Por Covadonga López Alonso y María Matesanz del Barrio
}

López Alonso, Covadonga y Matesanz del Barrio, María (eds.) (2009). Las plataformas de aprendizaje: del mito a la realidad. Madrid, Biblioteca Nueva. 258 páginas. ISBN: 978-84-9742-944-3.

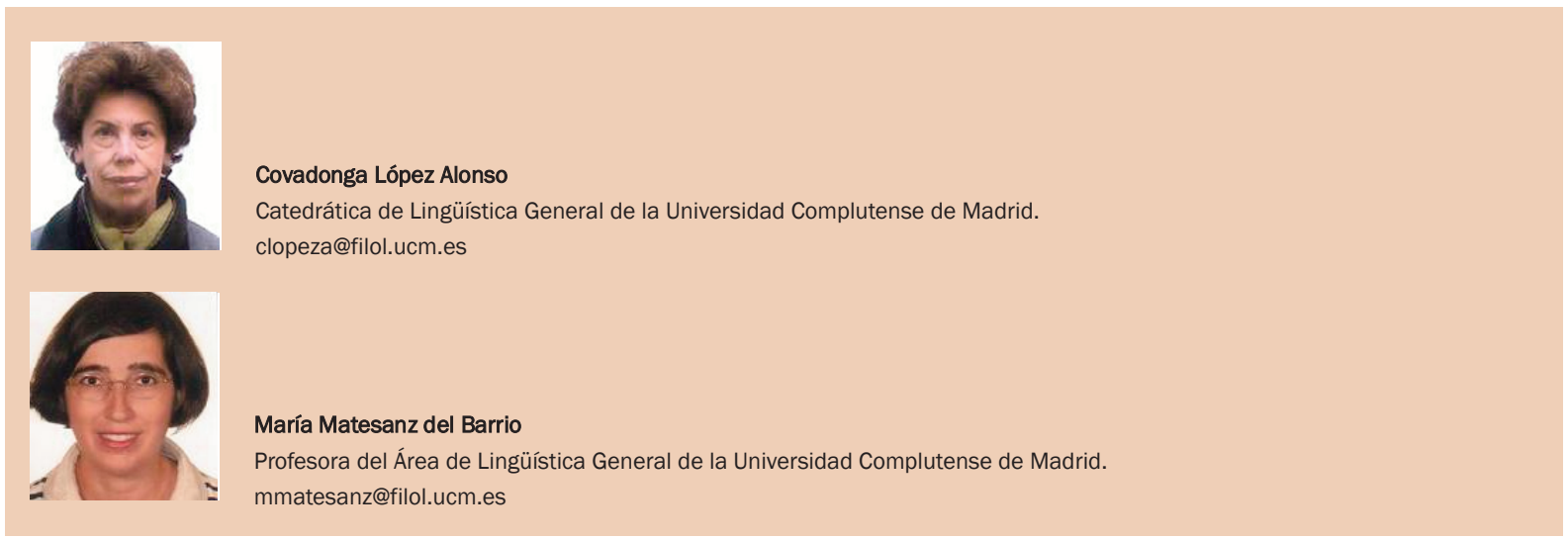

| Fecha presentación: 25/09/2009

| Aceptación: 23/10/2009

I Publicación: 04/12/2009

La rapidez con la que hemos asimilado el aprendizaje en plataformas educativas casi nos ha hecho olvidar que hemos dado este paso de gigante en poco más de una década. No son muchos los mitos que, en el ámbito de la educación, han caído de forma tan rápida y silenciosa y han devenido una realidad presente en todos los niveles de enseñanza. Este es el punto de partida de los estudios y reflexiones reunidos en Las plataformas de aprendizaje: del mito a la realidad, un libro novedoso porque conjuga presente y futuro en Educación Superior, tanto en enseñanzas no presenciales -e-learning- como mixtas -b-learning-, con el objetivo de mejorar la calidad de los aprendizajes en contextos pedagógicos concretos y evaluar la rentabilidad de diferentes modelos.

El cambio metodológico que están abordando las universidades en Europa, como consecuencia de la introducción de nuevos enfoques educativos y la implantación de las nuevas tecnologías en el ámbito docente, obliga a apoyar y asumir nuevas formas de entender la construcción y comunicación del conocimiento. En particular, la implantación de tecnologías específicas del ámbito docente, como son los campus virtuales, hace necesaria la compatibilidad de métodos y tecnologías. La especificidad de las enseñanzas universitarias lleva a la creación de espacios metodológicos apropiados a las necesidades de alumnos y profesores.

\section{Emergencia de las tecnologías en la Educación} Superior

Las tecnologías por sí mismas no son un recurso de aprendizaje y comunicación de conocimiento y, por tanto, es necesario realizar un trabajo de integración en una acción educativa, en función de cuáles sean las finalidades de la actividad, qué sujetos van a utilizarlas, desde qué contextos de trabajo, o qué requerimientos técnicos se necesiten. Identificar el para qué, cómo y cuándo de su uso y sus diferentes alternativas determinará los logros de los objetivos educacionales. Una propuesta híbrida de comunicación y técnica exige una interrelación entre usos sociales y tecnologías, ya que el contenido de un mensaje se actualiza en determinados géneros discursivos que, a su vez, toman forma, se transportan y contextualizan en el medio informático; las modalidades de actualización de la palabra no dependen exclusivamente de los géneros comunicativos sino también de los medios y, en este sentido, la politipología de la voz en Internet tolera al usuario pasar de un género a otro en una interrelación constante. Una aplicación didáctica con estos medios implica movilizar planteamientos teóricos adecuados, diseñar estrategias metodológicas que permitan potenciar usos efectivos de 
las TIC (Tecnologías de la Información y de la Comunicación), preguntarse qué hacen los estudiantes con esos dispositivos de información y de comunicación, o cuál es el impacto de esas tecnologías.

Las transformaciones sufridas en la Educación Superior son el resultado de una evolución de los modelos y métodos pedagógicos, especialmente en las últimas décadas. Esas pedagogías, marcadas por los objetivos, exigen métodos y medios que gestionen la búsqueda de la información, faciliten una autonomía de los saberes, fomenten la capacidad crítica y el autoaprendizaje, y respondan a las necesidades socioculturales. Ante esos nuevos imperativos, se da entrada, de forma expresa, a los multimedia y a las TIC, y se desarrollan procedimientos de creación del saber a partir de las redes complejas de los hipertextos, que reflejan el acerbo cultural y de conocimientos de un mundo marcado por una conciencia colectiva compartida. Desde un planteamiento socioconstructivista, esos novedosos estilos de información necesitan, también, métodos de trabajo diferentes, ya sean individuales, en grupo o tutorados, aprendizajes en situación que posibilitan tipos distintos de conocimientos, ya que los sujetos no sólo reciben una información general sino que construyen sus propios saberes, como resultado de procesos cognitivos individuales con los que asimilan las informaciones y las aplican a contextos plurales. Las tecnologías, por ello, refuerzan la actividad formativa de los estudiantes según sus capacidades, experiencias y deseos de aprender. Las TICE necesitan modelos pedagógicos que, rompiendo con estilos anteriores, den respuestas individualizadas según el tipo de aprendientes y sus necesidades formativas; para ello, hay que precisar, al menos, los tres parámetros siguientes: (i) el objeto de información, (ii) cómo adquirir competencias que formen al sujeto y le capaciten para una inserción laboral y un aprendizaje a lo largo de la vida, y (iii) de qué manera lograr la formación necesaria, apoyándose en enseñantes, procesos y medios. La utilización de las TICE implica una planificación diversificada de los actantes educativos y, a su vez, el modelo pedagógico que se elija debe fijarse en los cuatro elementos siguientes: (i) objetivos del aprendiente, (ii) finalidad de la enseñanza, (ii) entorno de aprendizaje, y (iv) necesidades y respuestas sociales.

Desde esos enfoques teóricos, flexibles, abiertos, tolerantes, y centrados en el alumno, las tecnologías y soportes electrónicos (i) se configuran como nuevas formas de presentar la información, almacenarla, y acceder al conocimiento, (ii) facilitan la aplicación de aprendizajes diferenciados, (iii) son instrumentos cognitivos que hacen posible la construcción de saberes, y (iv) agilizan la adquisición de conocimientos en una formación continua, ya que posiblemente, según las necesidades personales y laborales de los sujetos, vayan a seguir utilizando las TICE a lo largo de su vida.

Las tecnologías representan, por ello, un nuevo paradigma de enseñanza, marco innovador de información y comunicación que debe apoyarse en presupuestos cognitivos y socioconstructivistas, de modo que el aprendizaje, sea cual sea la disciplina o materia, consolide la interacción equilibrada de los parámetros pedagógicos de sujeto/objeto/agente, lográndose así una asimilación de los conocimientos basada en la interrelación de esos tres ejes.

\section{Aprendizaje y comunicación del conocimiento en Lingüística}

En el marco esbozado hemos desarrollado una metodología que planifica las relaciones de aprendizaje y que se articula en dos fases: (i) apropiación efectiva de la tecnología, y (ii) innovación en la concepción del trabajo en consonancia con la tecnología.

La innovación didáctica y sus estrategias de aprender se fundamentan en una ergonomía cognitiva en donde las prácticas realizadas son una prolongación de las capacidades cognitivas de los sujetos; el aprendizaje, de este modo, es el resultado final de un proceso, de ahí la importancia que cobra la construcción de los conocimientos. El plan de acción se centra en: (i) la representación de las tareas que van a elaborarse -informaciones que deben retenerse, datos que tienen que analizarse, conceptos que hay que relacionar, problemas que exigen soluciones etc.-; (ii) los medios con los que se cuenta; y (iii) capacidad de generar otros nuevos saberes. La conciencia de los propios recursos -metacognición- unida, además, al contexto tecnológico que se erige como espacio comunicacional e instruccional, induce a nuevas posibilidades de interrelación por el solo hecho de trabajar en un entorno cognitivo y con una red de usuarios.

Esta metodología ha propiciado la creación del entorno E-Ling, en el que el estudiante es el responsable de la construcción de su propio conocimiento, favoreciéndose, así, un aprendizaje significativo a partir de la estructura conceptual que el sujeto ya posee. No se trata, únicamente, de una virtualización de enseñanza previa en la que el estudiante lee en la pantalla lo que ha podido leer en papel -e-reading-, sino que es un medio de: (i) adquirir conocimientos y habilidades en Lingüística, y (ii) comunicarse, articulándose todos los saberes en una interacción e interactividad entre profesor(es) y estudiante(s) y estudiantes entre sí. Las interacciones y el acceso a las informaciones, además, se dan de forma síncrona o asíncrona, lo que proporciona una gran elasticidad y nuevos roles en las relaciones de enseñanza. Este entorno plural de comunicación es innovador, ya que se han diseñado estrategias específicas b-learning y metodologías dinámicas con el fin de lograr un aprendizaje efectivo de la Lingüística, tanto en la enseñanza general como en la iniciación a la investigación. El docente virtual E-Ling, a su vez, se sitúa en otro espacio formativo en el que guía al estudiante y, de esta forma, las interacciones entre ambos y el objeto de estudio son la base del aprendizaje. El estímulo para el aprendizaje es excelente no sólo por la organización de los contenidos que responden a: (i) la calidad y cantidad de la información, (ii) estructuración de los contenidos, (iii) interactividad, y (iv) adecuación a los objetivos y posibilidades de los estudiantes, sino también por la riqueza que supone el servirse de modelos síncronos y asíncronos, dependiendo de las herramientas y géneros electrónicos que se utilicen. Esta enseñanza combinada significa un cambio de paradigma en la educación universitaria, renovación relacionada con: (i) el triángulo profesor/estudiantes/objeto de conocimiento, y (ii) la combinación de metodologías de enseñanzas presencial y virtual.

La propuesta de cuatro escenarios didácticos en el espacio E-ling -contenidos, actividades, técnicas y herramientasque están presentes en todas las experiencias son los medios de los que dispone el alumno para generar su propio conocimiento. El modelo cognitivo teórico desarrollado en ELing, que integra docencia, investigación y TIC, ha sido validado mediante diversos casos de estudio realizados en 
un entorno b-learning. Durante tres cursos seguidos (20052008), hemos trabajado en varias asignaturas con la metodología desarrollada con E-Ling, en cuatro aspectos complementarios: 1) aprender a investigar; 2) investigar para aprender; 3) aprender colaborativamente; 4) consolidar el aprendizaje, último foco de interés abierto. Las experiencias englobadas en las tres primeras líneas están encaminadas al desarrollo de metodologías que permitan al alumno adquirir conocimientos a través de la investigación, construyendo de forma colaborativa conocimientos que, después, son compartidos y comunicados al grupo; la cuarta línea de trabajo está orientada a analizar formas de consolidar individualmente los conocimientos adquiridos. Todas las experiencias realizadas con la metodología de E-Ling han sido evaluadas y han demostrado que mejoran la calidad del aprendizaje, principalmente de la investigación, a la vez que favorecen las colaboraciones a distancia y los intercambios e interacciones entre los usuarios.

Por todo ello, el modelo propuesto permite aprendizajes y desarrollos personales y sociales interactivos en el que se enlazan, de forma armoniosa tres parámetros: (i) qué aprendemos -Lingüística-, (ii) cómo aprendemos -cooperativa y colaborativamente- y (iii) dónde aprendemos -enseñanza presencial y espacio virtual E-Ling. Además, la metodología desarrollada no sólo supone un cambio de paradigma en la Enseñanza Superior, sino que es aplicable a otras áreas de conocimiento, lo que incrementa el interés de la investigación realizada.

\section{Descripción de la estructura del libro}

El libro está organizado en tres partes. La primera parte Las plataformas de formación- consta de tres capítulos, en los que se analizan algunos aspectos concretos de las plataformas de aprendizaje dirigidas a estudiantes universitarios, con especial atención a las interacciones y tipo de comunicaciones que se realizan a través de ellas. La segunda parte -Aprender y comunicar en una plataforma b-learning- la constituyen cuatro capítulos que comparten el mismo hilo conductor, la propuesta de metodologías basadas en modelos teóricos cognitivos de aprendizaje que permiten la creación de un espacio virtual que integra docencia, investigación e innovación, el espacio virtual E-Ling. La tercera parte del volumen -Interacciones en las plataformas elearning- se centra en las relaciones de comunicación que entablan los usuarios de los espacios virtuales de formación. Las interacciones entre ellos son diversas y dan lugar al establecimiento de estrategias, constitutivas de tipología en la actuación en línea.

El recorrido que se propone al lector intenta dar respuesta a las preguntas que puede hacerse cualquier usuario de estos soportes: qué son, cómo son, cómo funcionan y, sobre todo, qué aportan estos entornos virtuales en ámbitos universitarios, en particular ante el cambio de mentalidad que conlleva el nuevo Espacio Europeo de Educación Superior. 